\title{
A Coupled/Uncoupled Computational Scheme for Deformation and Fatigue Damage Analysis of Unidirectional Metal-Matrix Composites
}

REFERENCE: Wilt, T. E., Arnold, S. M., and Saleeb, A. F., "A Coupled/Uncoupled Computational Scheme for Deformation and Fatigue Damage Analysis of Unidirectional MetalMatrix Composites," Applications of Continuum Damage Mechanics to Fatigue and Fracture, ASTM STP 1315, D. L. McDowell, Ed.. American Society for Testing and Materials, 1997, pp. $65-82$.

\begin{abstract}
A fatigue damage computational algorithm utilizing a multiaxial, isothermal, continuum-based fatigue damage model for unidirectional metal-matrix composites has been implemented into the commercial finite element code MARC using MARC user subroutines. Damage is introduced into the finite element solution through the concept of effective stress that fully couples the fatigue damage calculations with the finite element deformation solution. Two applications using the fatigue damage algorithm are presented. First, an axisymmetric stress analysis of a circumferentially reinforced ring, wherein both the matrix cladding and the composite core were assumed to behave elastic-perfectly plastic. Second, a micromechanics analysis of a fiber/ matrix unit cell using both the finite element method and the generalized method of cells (GMC). Results are presented in the form of $S$ - $N$ curves and damage distribution plots.
\end{abstract}

KEYWORIS: continuum damage mechanics, cracking, fatigue (materials), fracture (materials), metal-matrix composites, coupled deformation damage. uncoupled deformation damage, finite element methods

In advanced engine designs, materials that allow higher operating speeds and longer durability in addition to decreased weight are desirable. The use of metal-matrix composites (MMCs) may provide these benefits. For example, titanium metal-matrix composite (TMC) rotors are projected to have significant benefits in terms of increased rotor speeds and lower weight, as compared to the nickel and titanium rotors currently in service. However, to fully realize the benefits offered by MMCs, computationally efficient design and life prediction methods must be developed. Analysis of typical aerospace structures subjected to complex thermomechanical load histories requires the use of computational approaches such as the finite element method. In this regard, it is desirable to develop a life prediction algorithm that can be used in conjunction with the finite element method.

Historically, two basic approaches have been used in predicting the life of structures; uncoupled or fully coupled deformation-damage methods. A typical uncoupled analysis consists of obtaining the stress state for each element from a finite element analysis, and then, using the stress state data as input to a fatigue damage model, the number of cycles to the initiation of a crack are predicted. Subsequently, a "local" fracture mechanics approach is then used to propagate the crack. For example, a new finite element mesh is constructed to model the crack-

\footnotetext{
' Research engineer, University of Akron, Akron, OH 44325.

${ }^{2}$ Research engineer, NASA Lewis Research Center, Cleveland, OH 44135

${ }^{3}$ Professor, Civil Engineering. University of Akron, Akron, OH 44325.
}

NASA/TM. 
tip zone using a series of double nodes. The propagation of the crack is then controlled by a strain energy release rate criteria in conjunction with a node release scheme.

An alternative to the uncoupled method is a fully coupled deformation and damage method By utilizing the concept of effective stress/strain equivalence, the effects of damage are accounted for in the finite element solution. Through the degradation of the material properties, as will be described in the computational scheme section, for a specified increment in damage, the individual finite element material properties are degraded and the subsequent finite element analysis then calculates the corresponding stress redistribution caused by the damage. This sequence of material degradation (representing damage) followed by re-analysis to capture stress redistribution effects is repeated until structural failure.

This is the approach taken in the present study. Specifically, the computational scheme developed uses MARC, a nonlinear finite element code in which the fatigue damage algorithm is coupled to MARC through the use of provided user subroutines. In the next section, the requisite fatigue damage equations are presented. In the following section, the computationally coupled fatigue damage algorithm will be outlined. Finally, two applications of the computational scheme will be presented. The first example is a reinforced MMC ring, representing a typical engine component, and the results will be presented in terms of the evolution of damage in the ring cross section. The second example is a micromechanics analysis of a fiber-matrix representative volume element (RVE). The damage distribution in the matrix will be shown.

\section{Fatigue Damage Formulation}

The fatigue damage calculations utilize a recently developed multiaxial, isothermal, continuum damage mechanics model for the fatigue of unidirectional metal-matrix composites $|/|$. The model is phenomenological, stress-based, and assumes a single scalar internal damage variable. Note that for an initially anisotropic material, the evolution of the damage, although a scalar, is directionally dependent. As will be shown, this directional dependence is accounted for in the terms, $\hat{F}_{m}, \Phi_{f l}$, and $\Phi_{u}$. The present multiaxial, isothermal, continuum damage model for unidirectional metal-matrix composites may be expressed as $[I]$

$$
\int_{n_{k},}^{D_{k}} d D=\int_{0}^{N}\left[1-\left.(1-D)^{\beta+1}\right|^{\alpha}\left[\frac{\hat{F}_{m}}{1-D}\right]^{\beta} d N\right.
$$

$N$ is the number of cycles at the current stress state $\left(\sigma_{k}\right)$ and the increment in damage, $\left(D_{k}-\right.$ $D_{k-1}$ ), where $D_{k}$ and $D_{k-1}$ is the amount of damage at the current and previous increments. respectively. The quantity, $\alpha$, that is a function of the current stress state is defined as

$$
\alpha=1-a \frac{\left\langle\Phi_{f l}\right\rangle}{\left\langle\Phi_{u}\right\rangle}
$$

where \langle\rangle are the Macauley brackets. In $\mathrm{Eq}_{2} 2$, the fatigue limit surface, $\Phi_{j l}$, and the static fracture surface, $\Phi_{w}$, are defined as

$$
\begin{gathered}
\Phi_{n t}=\frac{1}{2} \frac{\max }{t_{0}} \frac{\max }{t} F_{(j)}\left(\sigma_{i j}(t)-\sigma_{i j}\left(t_{0}\right)\right)-1 \\
\Phi_{u}=1-\frac{\max }{t} F_{(\omega)}\left(\sigma_{i j}(t)\right)
\end{gathered}
$$

Note, the case, $\left\langle\Phi_{u}\right\rangle=0$, indicates static fracture, which is failure, making it unnecessary to perform the fatigue calculations. Thus, having to consider the possibility of $\alpha$ being undefined 
is unnecessary. As will be discussed in the following section, the finite element is considered to have failed completely. The case, $\left\langle\Phi_{n}\right\rangle=0$, indicates that the current stress state is below the fatigue limit and thus $\alpha$ is set equal to 1 . This presents a special case when integrating the fatigue damage expression, Eq 1, and will be considered later in this section.

The quantity, $\hat{F}_{m}$, the normalized stress amplitude, is defined as

$$
\hat{F}_{m}=\frac{1}{2} \frac{\max }{t} \frac{\max }{t_{0}} F_{(m)}\left(\sigma_{i j}(t)-\sigma_{i j}\left(t_{0}\right)\right)
$$

In the preceding equations, $t_{10}$ is the time at the beginning of the current load cycle, and $t$ is some time during the load cycle. The general form for $F_{(f t),(u),(m)(m)}$ may be expressed as

$$
F_{1}=\sqrt{\frac{1}{()_{1}^{2}}\left\{\left(4 \omega_{1}^{2},-1\right) I_{1}+\frac{4 \omega_{1}^{2},-1}{\eta_{1}^{2}} I_{2}+\frac{9}{4} I_{3}\right\}}
$$

It is here in Eq 6 where the evolution of the damage becomes directionally dependent. This simply amounts to the assumption of partial anisotropy, where the "extent" (magnitude) of damage is affected by the directionality of the stress state. Specifically, the directional dependence enters through the quantities, $I_{1}, I_{2}, I_{3}, \omega_{1}$, and $\eta_{1}$. The quantities, $I_{1}, I_{2}, I_{3}$, are invariants having the form

$$
\begin{aligned}
& I_{1}=\frac{1}{2} S_{i j} S_{i j}-d_{i} d_{j} S_{j k} S_{k i}+\frac{1}{4}\left(d_{i} d_{j} S_{i j}\right)^{2} \\
& I_{2}=d_{i} d_{j} S_{j k} S_{k i}-\left(d_{i} d_{j} S_{i j}\right)^{2} \\
& I_{3}=\left(d_{i} d_{j} S_{i j}\right)^{2}
\end{aligned}
$$

that are a function of the current deviatoric stress state, $S_{i j}^{k}$, as well as the vector, $d_{i}$, that defines the materials' fiber orientation. In addition, the terms, $\omega_{1}$, and $\eta_{k}$, represent the ratios of longitudinal to transverse normal and shear stresses, respectively. Note, the longitudinal direction is parallel to the fiber direction and transverse is perpendicular to the fiber direction. For initially transversely isotropic materials, $\omega_{t}$, and $\eta_{k}$, are $>1$, and for isotropic materials, $\omega_{i}$, and $\eta_{\ell}$, are $=1$.

For a current state of stress, $\sigma_{k}$, which is above the fatigue limit, that is, $\alpha \neq 1$ (integrating Eq I) results in an expression for the number of cycles, $N$, that is

$$
N=\frac{\left(\left[1-\left(1-D_{k}\right)^{\beta+1}\right]^{1-\alpha}-\left[1-\left(1-D_{k-1}\right)^{\beta+1}\right]^{1 \cdots \alpha}\right)}{\hat{F}_{m}^{\beta}(1-\alpha)(\beta+1)}
$$

Note that $D_{k-1}$ is the total amount of damage at the beginning of the load block and $D_{k}$ is the total amount of damage at the end of this load block.

In the present computational scheme, since the damage increment is controlled, both $D_{k}$ and $D_{k-1}$ are known. That is, $D_{k}=D_{k-1}+\Delta D$ where $\Delta D$ is the user-specified increment in damage. Thus, $\mathrm{Eq} 8$ is used to predict the increment in the number of cycles for each element, $N^{*}$, due to the increment in damage.

To calculate the number of cycles to failure for an initial damage amount, $D_{k-1}$, let $D_{k}=1$, which results in the following

$$
N_{F^{\prime}}=\frac{\left(1-\left[1-\left(1-D_{k-1}\right)^{\beta-1}\right]^{1-\alpha}\right)}{\hat{F}_{m}^{\beta}(1-\alpha)(\beta+1)}
$$


As will be shown in the following section, it is also necessary to rewrite Eq 8 in terms of the damage $D_{k}$, that is

$$
\left.D_{k}=1-\left(1-\left.\left\{11-\left(1-D_{k}\right)_{1}\right)^{\beta+1}\right|^{1} \alpha+(1-\alpha)(\beta+1) \hat{F}_{m}^{\beta} N\right\}^{\frac{1}{1-\alpha}}\right)^{\frac{1}{\beta+1}}
$$

Now, consider the case in which the current stress state is below the fatigue limit, that is, $\left\langle\Phi_{f l}\right\rangle=0$, which leads to $\alpha_{k}=1$. Thus, Eq 1 takes the form

$$
\int_{n_{1}, 1}^{D_{1}} \frac{(1-D)^{\beta}}{1-(1-D)^{\beta+1}} d D=\int_{0}^{N} \hat{F}_{m}^{\beta} d N
$$

Upon integrating Eq 11 , the increment in cycles, $N$, with initial damage, $D_{k} \quad 1$, may be expressed as

$$
N=\left(\frac{\log \left[1-\left(1-D_{k}\right)^{\beta+1}|-\log | 1-\left(1-D_{k}\right)^{\beta+1} \mid\right.}{\hat{F}_{m}^{\beta}(\beta+1)}\right)
$$

Alternatively, the following expression for the damage, $D_{k}$, may be expressed as

$$
\left.\left.D_{k}=1-\{1-1\}-\left(1-D_{k-1}\right)^{\beta+1} \mid \exp \left((\beta+1) \hat{F}_{m}^{\beta} N\right)\right\}\right\}_{k+1}^{1}+1
$$

For the number of cycles to failure, let $D_{k}=1$

$$
N_{F}=\frac{-\log \left[1-\left(1-D_{k-1}\right)^{\beta+1}\right]}{\hat{F}_{m}^{\beta}(\beta+1)}
$$

The effect of damage is included in the finite element stress analysis by utilizing the concept of "effective stress" [2] and the hypothesis of strain equivalence [3,4]. Thus, the effect of damage may be accounted for by either using the effective stress, that is, increasing the stress, or by simply degrading the elastic and plastic material properties to represent the material softening due to damage. Material softening is used in the present approach with the degraded elastic constitutive matrix calculated by

$$
[\bar{C}]=\left(1-D_{k}\right)[C]
$$

and similarly, the plastic material properties, for example, yield stress, $\sigma_{v}$, is given by

$$
\bar{\sigma}_{y}=\left(1-D_{k}\right) \sigma_{y}
$$

\section{Computational Scheme}

\section{General Framework}

The present version of the fatigue damage algorithm utilizes average quantities in the damage calculations. For example, the stresses for each integration point are determined and then all integration points are averaged to give one stress state for each element. All subsequent damage calculations use these average quantities. However, the program was written in a sufficiently 
general form so that all of the damage calculations may be performed at each integration point with minimal modifications. Specifically, all that is required is increased dimensions for various storage arrays. Note that, because of the scalar nature of the damage parameter used here, together with the assumptions previously discussed, the task of local (constitutive) level integration is simply reduced to the use of the "exact" closed-form expressions given in the preceding section

A flowchart of the developed life prediction scheme is shown in Fig. 1. The deformation analysis is the actual finite element run. First, note there are two levels of failure criteria checks; element level and structural level. The element level includes a static fracture surface check that is part of the fatigue damage model (Eq 4). Additional criteria may be included such as a check on total mechanical strains, etc. If an element violates one of these failure criteria, that element is considered to have "failed" and its damage, $D$, is set equal to the maximum amount of damage allowed. As shown in Fig. 1, for a coupled analysis, the damage calculations are terminated and a deformation analysis is rerun in order to account for the stress redistribution

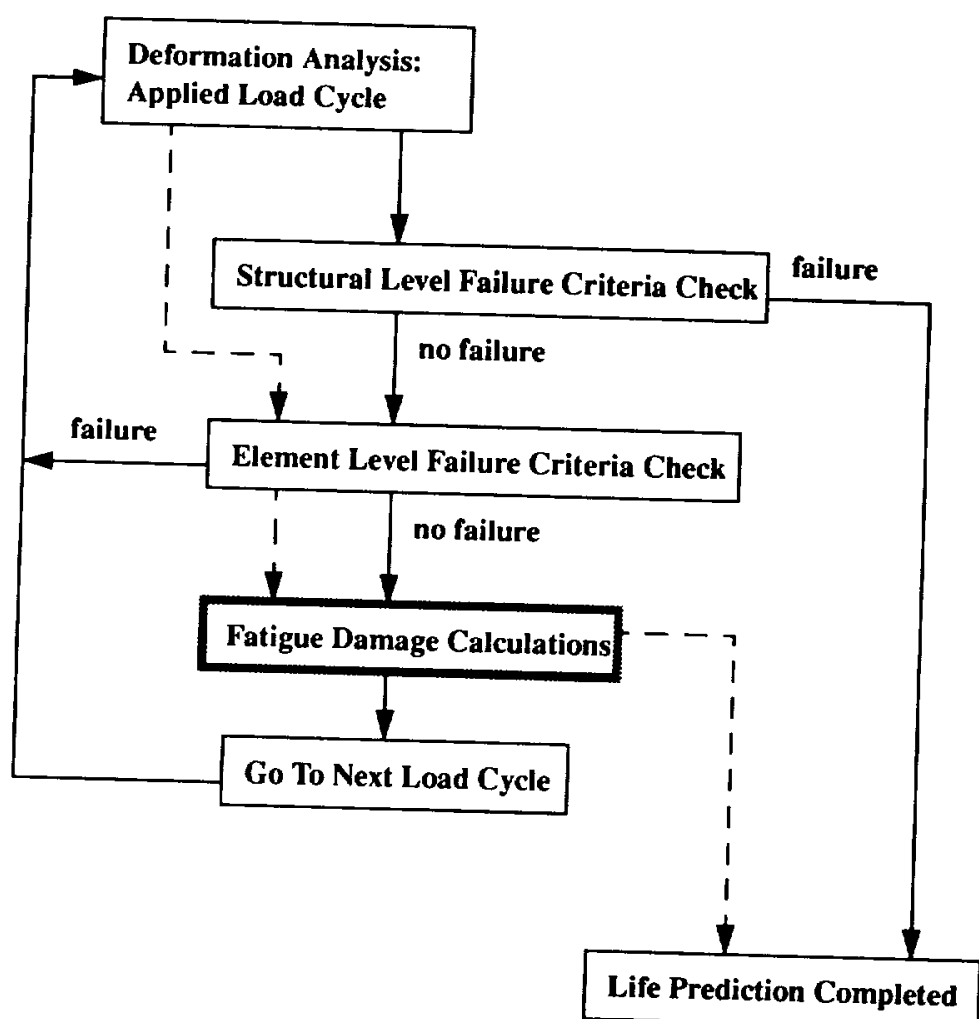

$---\rightarrow$ Uncoupled Analysis

$\longrightarrow \quad$ Coupled Analysis

FIG. 1-Coupled/uncoupled life prediction scheme. 
due to that element's failure. For an uncoupled analysis, when element failure occurs, the analysis does not loop back and perform another deformation analysis; instead, it continues to the next element and performs the damage calculations. The structural level criteria monitors the global response of the structure. This could take the form of a check upon selected nodal displacements that, if they violate a specified displacement criteria, the structure is considered to have failed. For example, the tip displacement of a turbine blade may be required to stay within a given tolerance. Again, note that for an uncoupled analysis no structural failure criteria check is performed since the present fatigue damage algorithm assumes that the structure, when subjected to the initial "applied" load cycle, is in a completely undamaged state.

The applied load cycles just mentioned are those actually defined by the user through the finite element input data file. In addition, the number of load increments per applied cycle must be specitied by the user. This is necessary so that the program can internally monitor when a given applied load cycle has been completed and begin the damage calculation phase. The number of load increments used in the load cycle usually depends on the nonlinearity of the structural response and requires experience on the part of the user. At each increment during the applied load cycle, the average stress (strain is optional) state for each element is stored. At the last load increment of the current applied load cycle, the DAMAGE subroutine is called to perform the fatigue damage calculations, see Fig. 2.

When DAMAGE is entered for each element, various element quantitics, such as $\alpha, \Phi_{l}, \Phi_{14}$, $\hat{F}_{m}$, are calculated and stored. When DAMAGE has been called for the last element in the mesh, the fatigue damage calculations are performed. Figure 2 shows the general algorithm for the fatigue damage calculations.

Presently, the damage calculations are controlled by the increment in damage, $\triangle D$. The user specities the allowable increment in damage, for example, $\Delta D=0.15,(15 \%)$. In CALCN, using $\mathrm{Eq} 9$ or $\mathrm{Eq} 12$, based upon the new value of damage and the given element's stress state, the number of cycles to failure, $N_{p}^{\prime}$, is calculated and stored. Next a "sorting" subroutine, SORTN, is called (see Fig. 2) to determine which element has the minimum number of cycles to failure and is chosen as the "controlling element," that is

$$
N_{\text {rimin }}=\min N_{r} \quad e=1 \rightarrow \text { numel }
$$

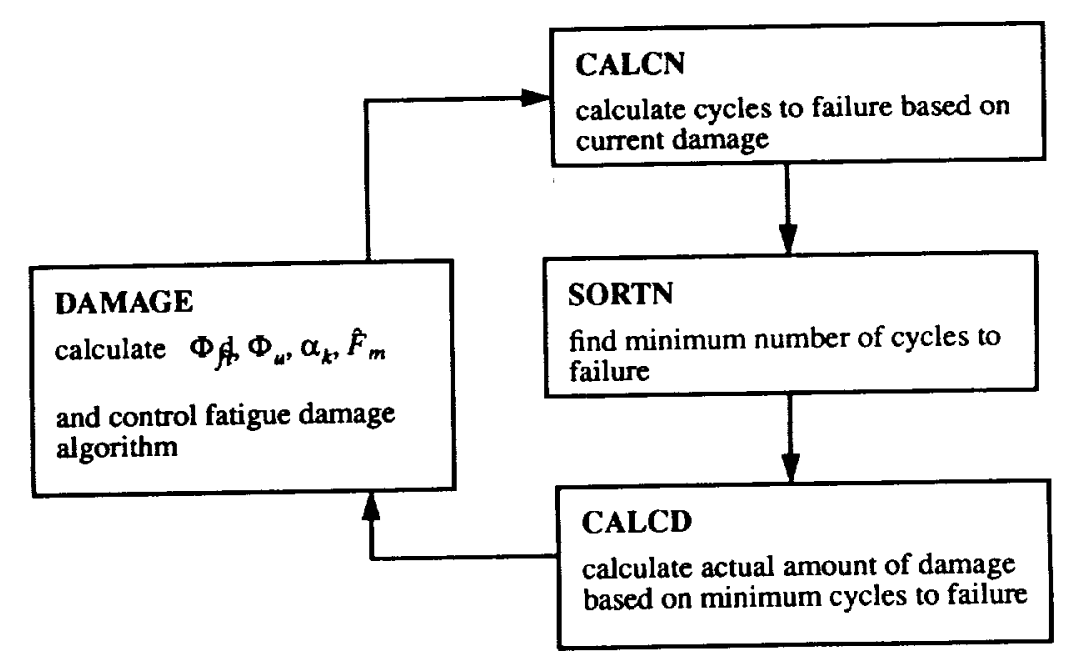

FIG. 2-Fatigue damage calculations. 


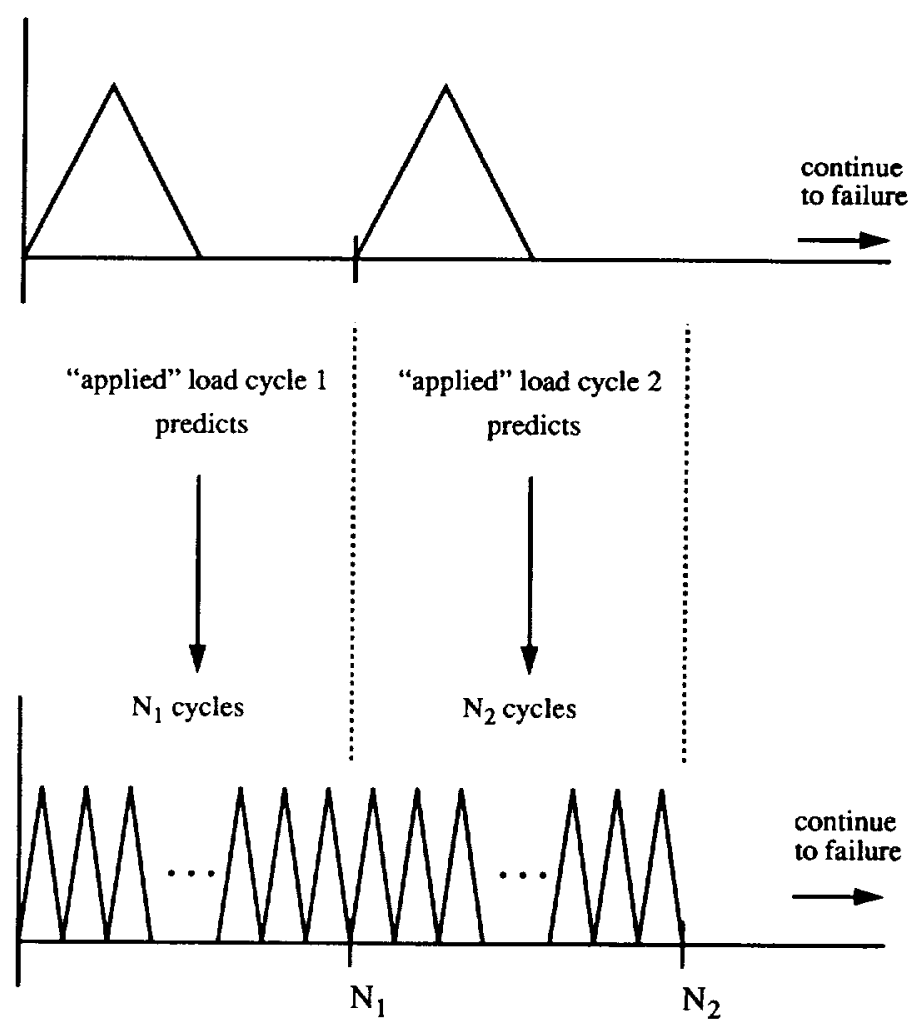

FIG. 3-Cycle scheme for a coupled analysis.

Once the controlling number of cycles has been determined, the corresponding, actual amount of damage, $D_{k}^{-}$, in all of the remaining elements must be recalculated. This is performed in subroutine CALCD using Eq 10 or 13. Note that since the damage was incremented by a specified amount, the controlling element's damage is already known.

Figure 3 shows the cycle scheme used in the code. Recall that the "applied" load cycle is the actual load history that is applied and used in the finite element analysis. The subsequent cycles, shown in dashed lines, are the predicted cycles corresponding to $N_{F \min }$ that is determined in SORTN. Here, it is assumed that the stress state in each element remains constant during the predicted $N_{\text {rimin }}$ cycles and at the end of $N_{F \text { min }}$, each element has incurred an amount of damage as calculated in CALCD. Note for a coupled analysis, the next applied load cycle is run in the finite element analysis to account for the stress redistribution due to the new damage state in each element (that is, $D_{k}^{c}$ ) and again a new $N_{F \text { min }}$ is predicted. This sequence of applied load cycle and predicted cycles is repeated until the structure has failed. For an uncoupled analysis, only one sequence, that is, only one applied load cycle, is performed. The resulting $N_{\text {rmin }}$ would be used to merely indicate the location of damage initiation.

In preparation for the subsequent applied load cycle, the element material properties are degraded according to the newly calculated element damage, $D_{k}^{\prime}$. Finally, a subroutine, PATSTR, was also written that generates PATRAN [5] element results files. These files contain damage distributions at specified increments during the fatigue damage analysis. In addition, output files containing a summary table of the current number of fatigue cycles and remaining 
cycles to failure for each element, and a summary table showing the damage evolution in each element are also generated.

Some general comments on the fatigue damage algorithm need to be made. First, once an element attains the user specified maximum allowable damage, the element is assumed to fail and is no longer considered in any subsequent damage calculations. In the example presented in the next section, a cutoff value of $95 \%$ was specified based upon preliminary experience with difficulty in achieving global convergence when the element stiffness was reduced below $5 \%$. Further investigation of convergence difficulties needs to be addressed. Second, in the present fully coupled damage-deformation analysis, a perfect plasticity model was used. This idealization eliminates the need to account for cyclic hardening, and the corresponding update of the internal variables, which may occur during a specific block of fatigue cycles. In order to accurately account for the hardening, a projection/update of the internal variables through the load block would be required. In addition, it is usually assumed that the fatigue damage calculations are applied to a "stabilized" stress redistribution. Thus, when hardening is present, more than one applied load cycle may be necessary in order to achieve the stabilized stress redistribution.

\section{MARC Implementation}

As an example, the present fatigue damage algorithm was implemented into the finitc element code MARC. Please note that based upon the discussion in the previous section, it should be apparent that the fatigue damage algorithm was developed in a form that is independent of the specific finite element code that is used. Most commercial finite element codes provide capabilities similar to those described here with regards to MARC.

MARC provides various user subroutines [6] that allow implementation of constitutive models, failure criteria, new elements, etc. By using a few select MARC user subroutines, the continuum-based fatigue damage model has been coupled with the nonlinear finite element solution scheme. The primary MARC user subroutine required is ELEVAR. In addition, when using the plasticity model contained in MARC, the subroutines HOOKLW and ANPLAS are used to degrade the elastic and plastic material properties, respectively. In this study, the user subroutine HYPELA was also used to implement the Bodner-Partom viscoplastic model into MARC.

The subroutine ELEVAR is called at the end of each load increment once global convergence has been attained, and is intended to be used to output element quantities at the end of a given increment. In this algorithm, ELEVAR is used to store the current converged stress state for each element during the applied load cycle. The meaning of applied load cycle is the same as discussed earlier in the previous section. This is done through the MARC subroutines HOOKLW, for the elastic constants, and ANPLAS, for the anisotropic yield stress ratios using Eqs 17 and 18. Again, HOOKLW and ANPLAS are used when the MARC plasticity model is used. For the Bodner-Partom viscoplastic model, similar calculations are performed within the HYPELA routine.

\section{Example Applications}

\section{A Cladded MMC Ring Insert}

As stated previously, one of the primary motivations of this research is to establish a computationally efficient method for predicting the fatigue life of typical aerospace components. This includes the ability to predict the location of damage initiation and to be able to track the propagation of damage throughout the structure. With this in mind, the fatigue damage algorithm was applied to a cladded MMC ring. The reasons for choosing this specific structure are 
TABLE 1-Material properties for plastic deformation model

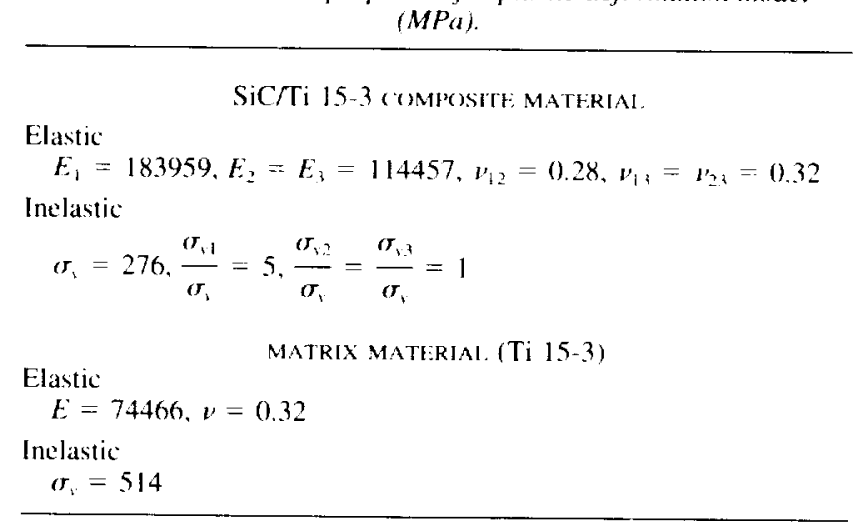
$(M P a)$.

Notr---The subscript, 1, denotes fiber direction.

twofold. First, it represents a MMC rotor insert currently under consideration in advanced engine designs. Secondly, because of its axisymmetric geometry and load conditions, qualitative stress distributions are known a priori. For example, maximum circumferential stress in the core occurs at its inner diameter and likewise for the cladding, thus providing some intuitive feel for where damage initiation will occur as well as how it may propagate.

The composite core was described by Hill's anisotropic elastic-plastic constitutive model available in MARC [0], while the matrix cladding was assumed to be isotropic and elastic perfectly-plastic. The elastic and inelastic material parameters required for the deformation analysis are given in Table 1, while the associated material parameters for the fatigue damage model are given in Table 2. Note that the matrix cladding utilizes the isotropic form of the fatigue damage model, that is, $\omega_{u}=\omega_{n}=\omega_{m}=\eta_{u}=\eta_{f t}=\eta_{m}=1$, whereas the composite core is represented by the transversely isotropic form of the model. The finite element model, representing the cross section of the ring, Fig. 4. consisted of 225 nodes and 64 eight-node axisymmetric elements (MARC element No. 28). A uniform pressure load was applied along the inner diameter of the ring.

With regards to the deformation analysis, burst pressure predictions have been previously made and compared to limited experimental data $[7,8]$. Very good correlation was observed. thus providing a level of confidence in the finite element modeling of the ring.

TABLE 2-Material properties for fatigue model [7] (MPa).

\begin{tabular}{|c|c|}
\hline \multicolumn{2}{|c|}{ SiCTTi $15-3$ COMPOSITE MATHRIAL. } \\
\hline$\sigma_{u}=10694$ & $\omega_{u}=5.5$ \\
\hline$\sigma_{f}=1972$ & $\omega_{n}=12.482$ \\
\hline$\beta=1.842$ & $\omega_{m}=11.8$ \\
\hline $\begin{array}{l}a=0.012 \\
M=22371\end{array}$ & $\eta_{\mu}=\eta_{n}=\eta_{m}=1.0$ \\
\hline \multicolumn{2}{|c|}{ MATRIX MATERIAL. (Ti 15-3)-ISOTROPIC SIMPLIFICATION } \\
\hline$\sigma_{u}=6081$ & $\omega_{u}=1.0$ \\
\hline$\sigma_{n}=965$ & $\omega_{n}=1.0$ \\
\hline$\beta=2.27$ & $\omega_{m}=1.0$ \\
\hline$a=0.0365$ & $\eta_{w}=\eta_{n}=\eta_{m}=1.0$ \\
\hline$M=6205$ & \\
\hline
\end{tabular}



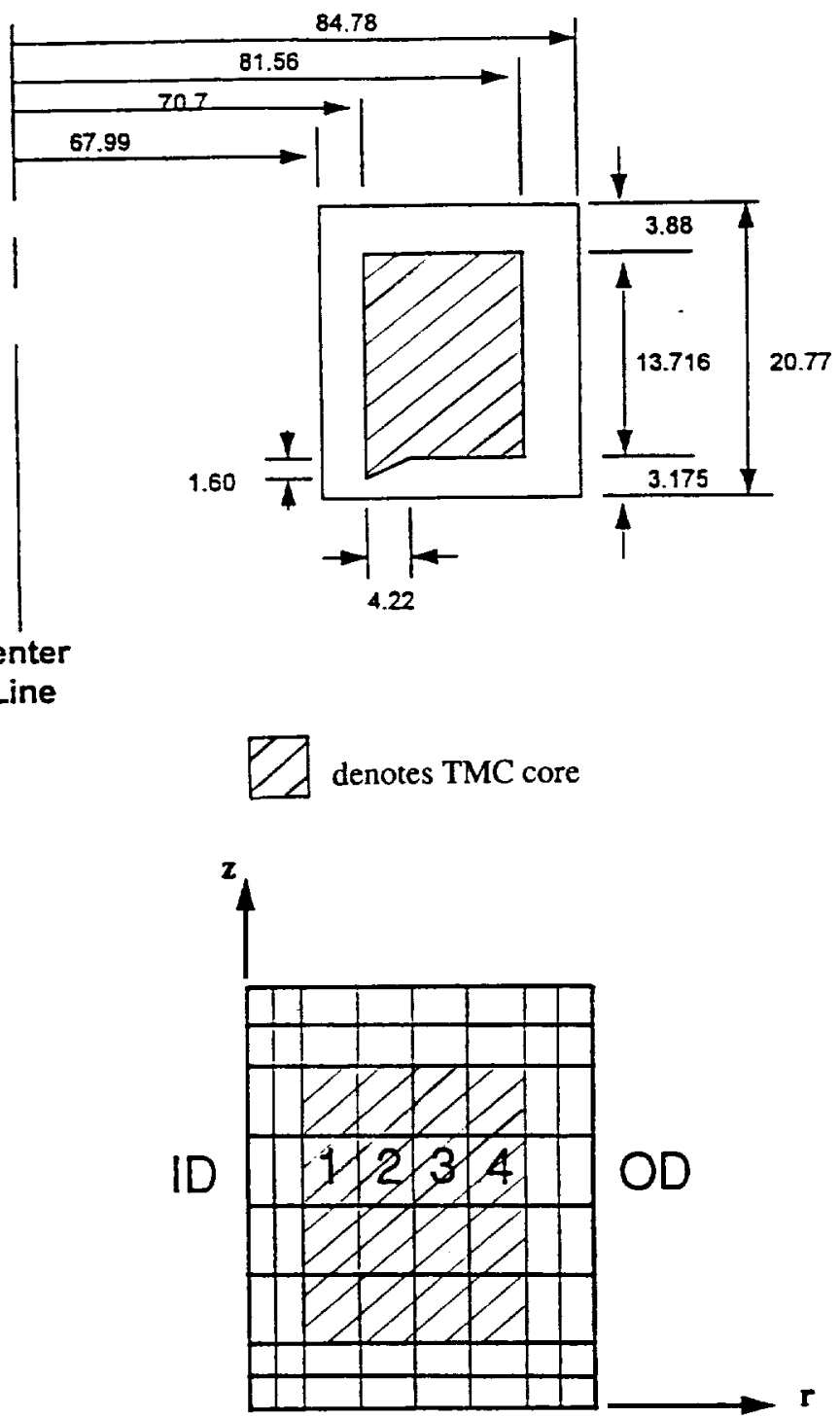

FIG. 4-Cladded MMC ring geometry and finite element model: (top) actual ring geometry (dimensions are in $\mathrm{mm}$ ) and (bottom) idealized ring geometry.

Two types of fatigue life analyses were performed, namely, an uncoupled and a coupled analysis. The uncoupled life prediction results were obtained by taking four elements, labeled $1,2,3$ and 4 in Fig. 4 (bottom), in the radial direction of the composite core of the ring. It was assumed that the stress state was relatively constant in the $z$-direction, thus one element would represent all of the elements in a column of the composite core. In the uncoupled analysis, no fatigue calculations were performed on the elements associated with the matrix cladding because of the initially low stress levels in the matrix cladding causing infinite fatigue lives to be 
calculated. A finite element analysis was run in which the ring was subjected to a cyclic pressure load. During the analysis, the stress state history for each of the four elements was stored. The stress history for a given element $(1,2,3$, or 4$)$ was then used to predict the corresponding fatigue life for that element. Since only a single cycle was used, no stress redistribution effects, due to damage, were accounted for in the uncoupled analysis. Figure 5 (top) shows the results of the uncoupled fatigue damage analysis. As expected, since Element 1 has the highest stress
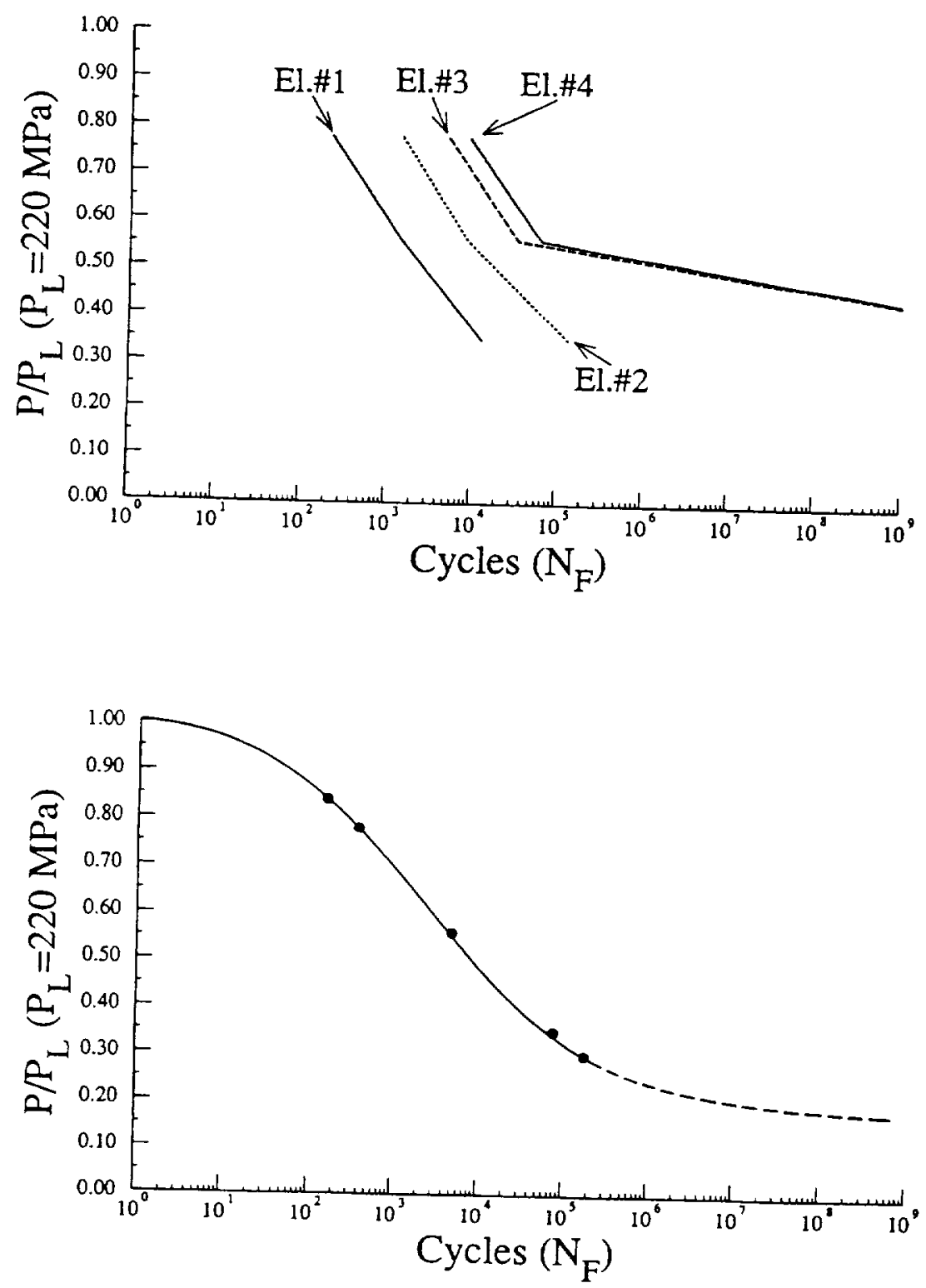

FIG. 5-Life prediction for uncoupled (top) and coupled (bottom) analysis. 
$N N_{F}=0.20$

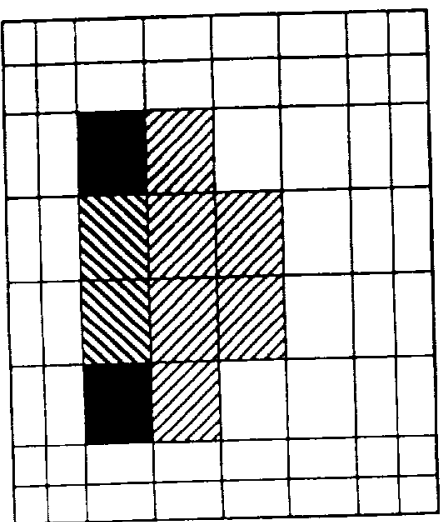

$N N_{F}=1$

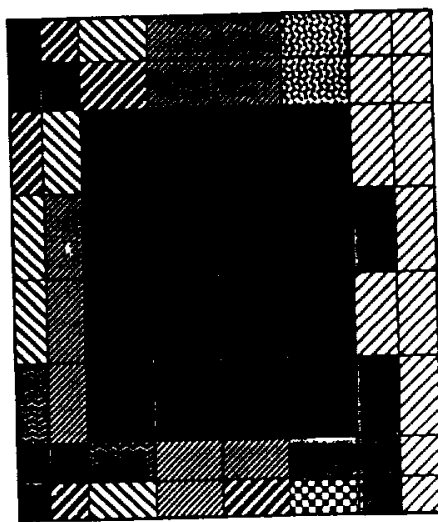

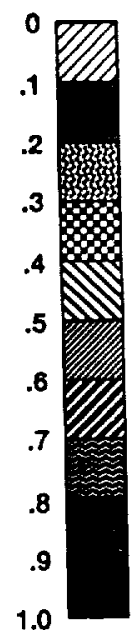

FIG. 6-Fatigue damage distribution in ring cross section: (left) $\mathrm{N} / \mathrm{N}_{\mathrm{I}} \approx 0.2$ and (right) $\mathrm{N} / \mathrm{N}_{\mathrm{Y}}=$ 1.0. Cycles to failure. $\mathrm{N}_{1}=155$.

state, it correspondingly has the shortest fatigue life. Thus, damage is predicted to initiate along the composite core inner diameter.

The fully coupled life prediction analysis follows the algorithm presented in the previous section that takes into account the effects of stress redistribution due to the propagation of damage. Now in the coupled analysis, finite lives and damage are predicted for the cladding, due to stress redistribution effects. Figure 5 (bottom) shows the fully coupled deformation and fatigue damage analysis results. The solid circles are analysis results obtained using the present fully coupled algorithm. A smooth solid line is drawn through these points to obtain the $S-N$ curve. Note the dashed part of the $S-N$ curve indicates an "extrapolation" since no analysis was run at a $P / P_{L}$ less than 0.30 . Upon comparison, one observes that at pressures close to the burst pressure, the fatigue life predicted by the coupled analysis is close to that of the uncoupled analysis, since at high stress levels, once the damage initiates in the core. "structural" failure of the ring occurred rapidly. On the other hand, at low stress levels, the fatigue life as predicted by the coupled analysis is longer than that predicted from the uncoupled analysis at the inside diameter. This difference may be viewed as the effect of propagation of the damage in the ring cross section. This propagation is caused by the stress redistribution effects that are automatically captured by performing a fully coupled deformation and fatigue damage analysis.

Finally, Fig. 6 shows two selected damage distribution plots in the ring cross section produced by the coupled fatigue damage analysis for $P / P_{L}=0.85$. Note that in Fig. 6 (left), the damage initiates along the inner diameter of the composite core. In addition, the first elements to completely fail are located in the inner radius "corners" of the TMC core. Therefore, the assumption used in the uncoupled analysis of a uniform stress state in the z-direction for a given column of core elements was not appropriate. Conversely, in Fig. 6 (right) structural failure of the ring is depicted (that is, the composite core has completely failed) and due to stress redistribution, the matrix cladding has accumulated significant amounts of damage. 

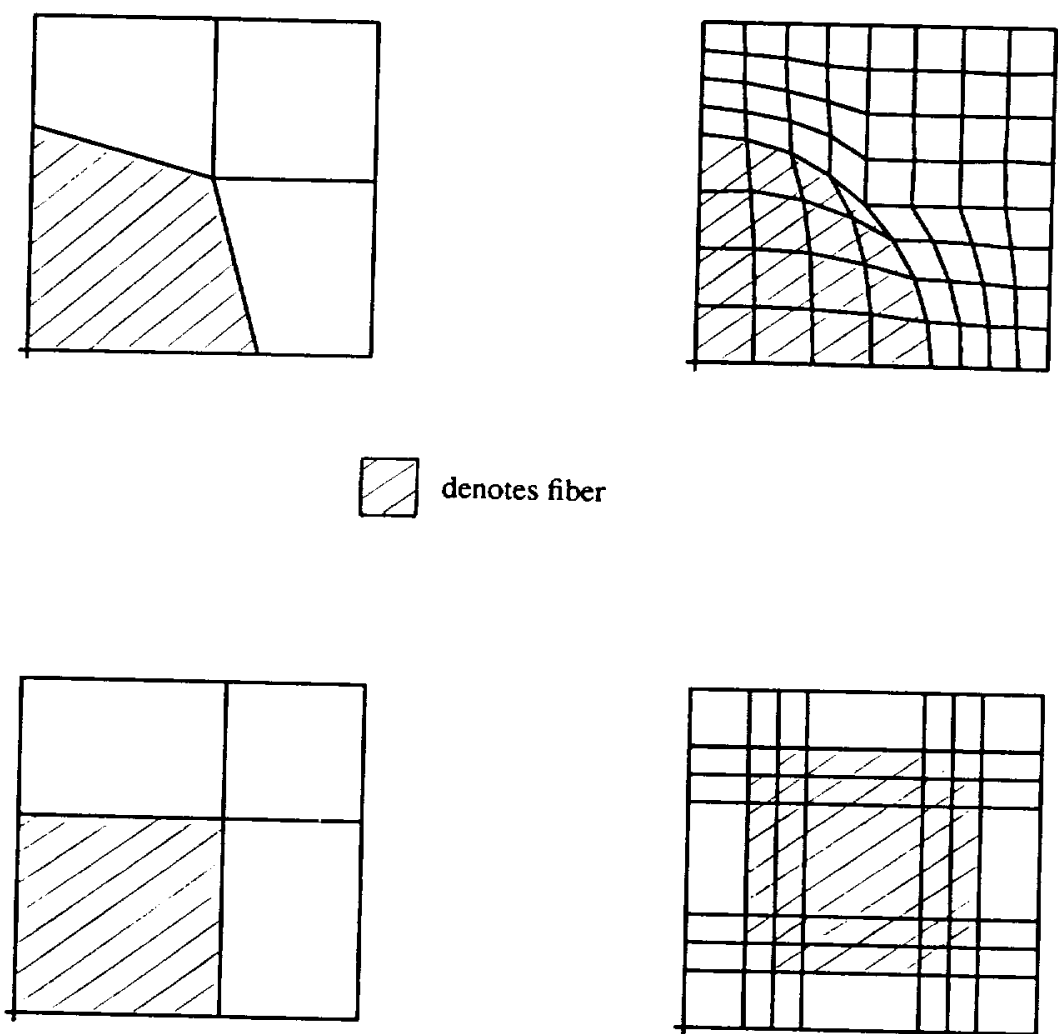

FIG. 7-Finite element mesh (top) and GMC (bottom) RVEs

\section{Micromechanical Failure Analysis}

The second application is on the microscale in which a fiber/matrix unit cell representing a square pack, 35\% fiber volume ratio (FVR), SiC/Ti-15-3 composite was analyzed. Two approaches were used to model and analyze the unit cell. The first approach utilized a finite element representation in which the unit cell was idealized using two mesh discretizations, consisting of 4 and 64 eight-node three-dimensional elements, Fig. 7. For the deformation response, the Bodner-Partom viscoplastic model was characterized for the Ti-15-3 matrix material (see Table 3) and the fiber utilized a simple isotropic linear elastic model (see Table 4).

TABLE 3-Material properties for viscoplastic deformation model (MPa) SiC fiber material.

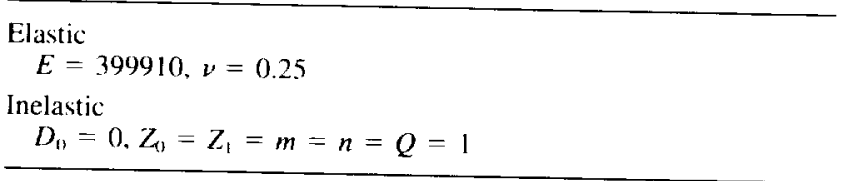


TABLE 4-Material properties for viscoplastic deformation model (MPa) Ti 15-3 matrix material.

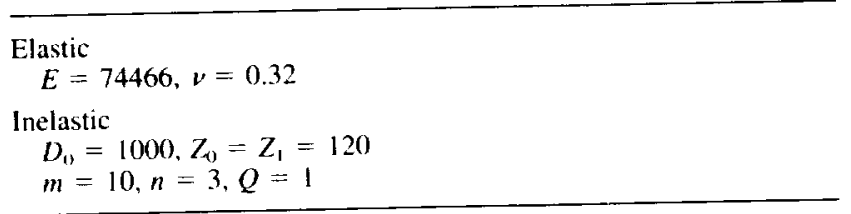

The Bodner-Partom viscoplastic model was implemented into MARC using the HYPELA user subroutine. The fatigue damage model, taken in its isotropic form, was characterized for the Ti-15-3 matrix constituent.

The second approach utilized the generalized method of cells, GMC, developed by Aboudi 19]. GMC is a continuum-based micromechanics model that provides closed-form expressions for the macro response of the composite in terms of the individual constituents (phases). The GMC model and the fatigue damage algorithm have been incorporated into the stand-alone micromechanics analysis code, MAC [/O]. MAC has the ability to analyze a material volume element subjected to various thermal, mechanical (stress or strain control), and thermomechanical load histories, and wherein different integration algorithms and a variety of constitutive models can be selected. Previous work $[I /]$ has shown the accuracy and computational efficiency of GMC with respect to deformation analysis. Part of this work, and future research, is to determine if GMC also provides accurate and computationally efficient fatigue damage predictions.

The analysis consisted of stress-controlled cyclic loads applied in the longitudinal direction with respect to the unit cell. All four unit cells (both finite element and GMC) produced identical

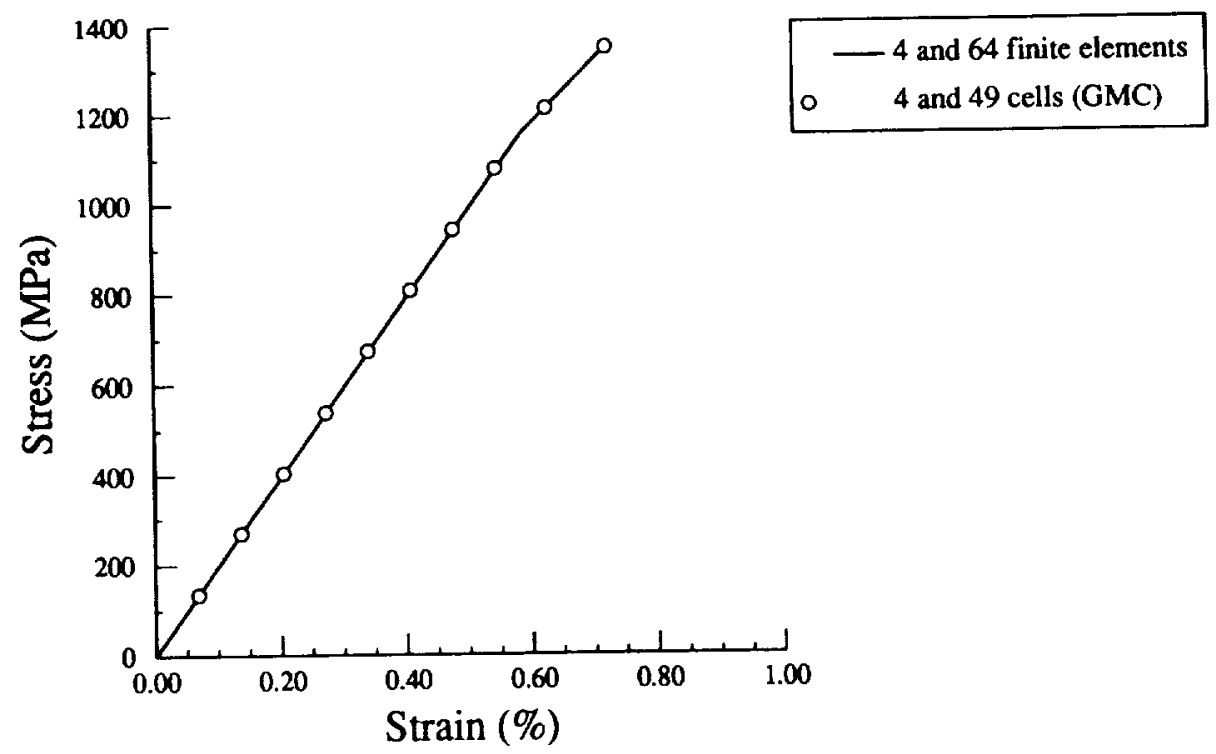

FIG. 8-Longitudinal stress versus strain response: finite element and GMC. 
TABLE 5-Fatigue lives for a 35\% fiber volume fraction SCS-6/Ti-15-3 composite loaded longitudinally.

\begin{tabular}{|c|c|c|c|c|c|c|}
\hline \multirow[b]{3}{*}{ Load, MPa } & \multicolumn{6}{|c|}{ Fatigue Life, Cycles } \\
\hline & \multicolumn{2}{|c|}{ Finite Element } & \multirow[b]{2}{*}{ Difference, $\%$} & \multicolumn{2}{|c|}{ GMC } & \multirow[b]{2}{*}{ Difference, $\%$} \\
\hline & 4 & 64 & & 4-Cell & 49-Cell & \\
\hline 1.345 & 2939 & 2858 & 3.0 & 2497 & 2494 & 0.2 \\
\hline 1207 & 4957 & 4859 & 2.0 & 4245 & 4234 & 0.25 \\
\hline 931 & 21497 & 20992 & 2.4 & 20459 & 20098 & 2. \\
\hline 793 & 64496 & 62366 & 3.4 & 61235 & 59843 & 2.3 \\
\hline
\end{tabular}

longitudinal macro stress-strain responses, as shown in Fig. 8. However, the predicted longitudinal fatigue lives are different, see Table 5. An example macrolongitudinal $S-N$ curve produced with the 64 finite element model is shown in Fig. 9. Note, that if the SCS-6 fiber is taken to have a constant ultimate tensile strength (UTS) value, the lower stress amplitude lives in Fig. 9 are run outs. Consequently, in order to agree with the experimentally determined fatigue lives, a fiber UTS model that degrades as a function of time and temperature is required, Fig. 10. All the lives indicated in Table 5 were generated using this time-dependent fiber UTS model. Referring to Table 5, note that the 4 and 64 finite element unit cells give results that are consistently within 2 to $3 \%$ of each other for all stress levels, and the 4 and 49 cell GMC results are within 0.2 to $2 \%$ of each other. This shows that for the longitudinal response the 4 finite element unit cell produces results that are comparable to the 64 finite element unit cell. Similarly, the 4-cell GMC representative volume element (RVE) gives results that are as accurate as the 49-cell GMC RVE. Table 6 shows a comparison between the 4 finite element unit cell versus the 4-cell GMC RVE and the 64 finite element unit cell versus the 49-cell GMC

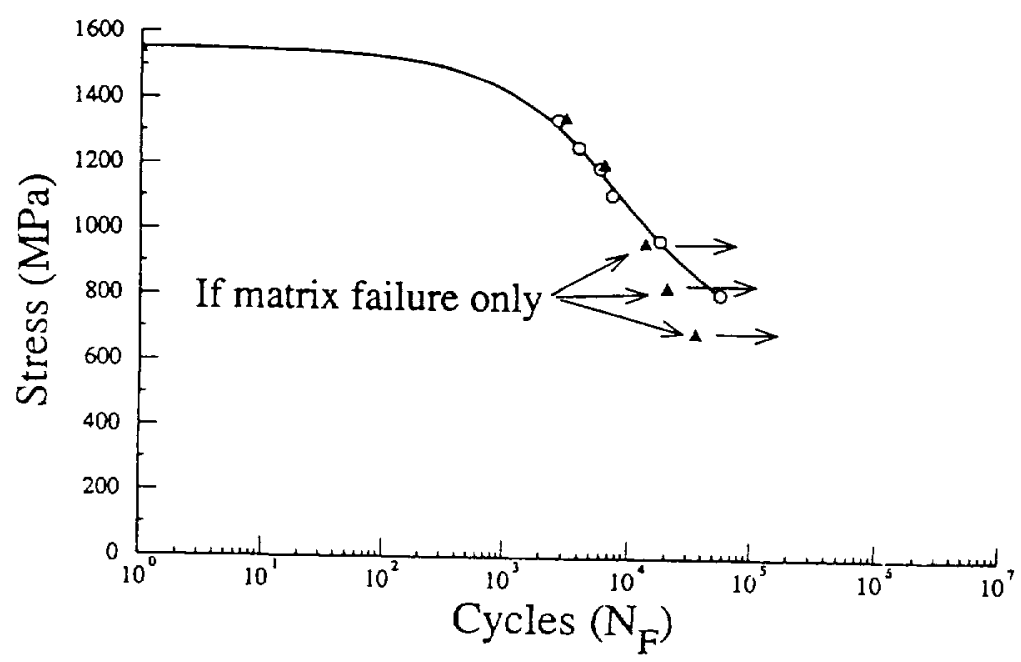

FIG. 9-Life prediction for SiC/Ti-15-3 composite. 


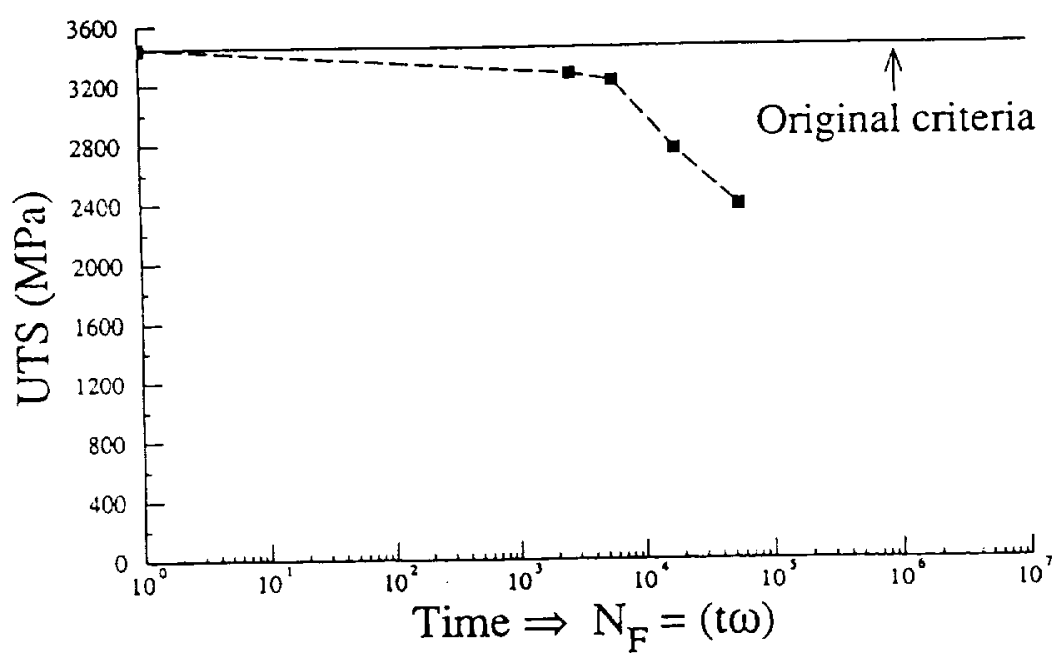

FIG. 10-Fiber constituent ultimate tensile strength (UTS) failure diagram

RVE. Note that at the higher stress amplitudes (1345 and $1207 \mathrm{MPa}$ ), the relative differences are on the order of 14 to $17 \%$, while at the lower stress amplitudes (931 and $793 \mathrm{MPa}$ ), there is a relative difference of 4 to $5 \%$.

Figure 11 shows the damage distribution for the fatigue analysis having a maximum stress amplitude of $1345 \mathrm{MPa}$. The damage distributions were taken just before failure of the unit cell (that is, fiber fracture). Figure 11 shows the corresponding damage distributions for both the 4 and 64 finite element unit cells and the GMC 4-cell and 49-cell RVE models.

\section{Conclusions}

A coupled/uncoupled deformation and fatigue damage algorithm has been presented. The algorithm utilizes a multiaxial, isothermal, stress-based, transversely isotropic continuum fatigue damage model in which the fatigue damage calculations are coupled with the nonlinear finite element solution using the concept of effective stress. Incorporated in the life prediction scheme are failure criteria checks at both the element and structural level. The algorithm has been applied to a cladded MMC ring insert representing a typical aerospace component and results have been presented in terms of $S-N$ curves along the damage distribution plots over the ring cross section. The fatigue damage results presented are qualitative in nature since no

TABLE 6-Fatigue lives for a 35\% fiber fraction SCS-6/Ti-15-3 composite loaded longitudinally.

\begin{tabular}{ccccccc}
\hline \multicolumn{7}{c}{ Fatigue Life, Cycles } \\
Load, MPa & $\begin{array}{c}\text { 4 Finite } \\
\text { Element }\end{array}$ & $\begin{array}{c}\text { 4-Cell } \\
\text { GMC }\end{array}$ & Difference, \% & $\begin{array}{c}\text { 64 Finite } \\
\text { Elements }\end{array}$ & $\begin{array}{c}\text { 49-Cell } \\
\text { GMC }\end{array}$ & Difference, \% \\
\hline 1345 & 2939 & 2497 & 17.7 & 2858 & 2494 & 14.5 \\
1207 & 4957 & 4245 & 16.7 & 4859 & 4234 & 14.7 \\
931 & 21497 & 20459 & 5.0 & 20992 & 20098 & 4.0 \\
793 & 64496 & 61235 & 5.3 & 62366 & 59843 & 4.0 \\
\hline
\end{tabular}



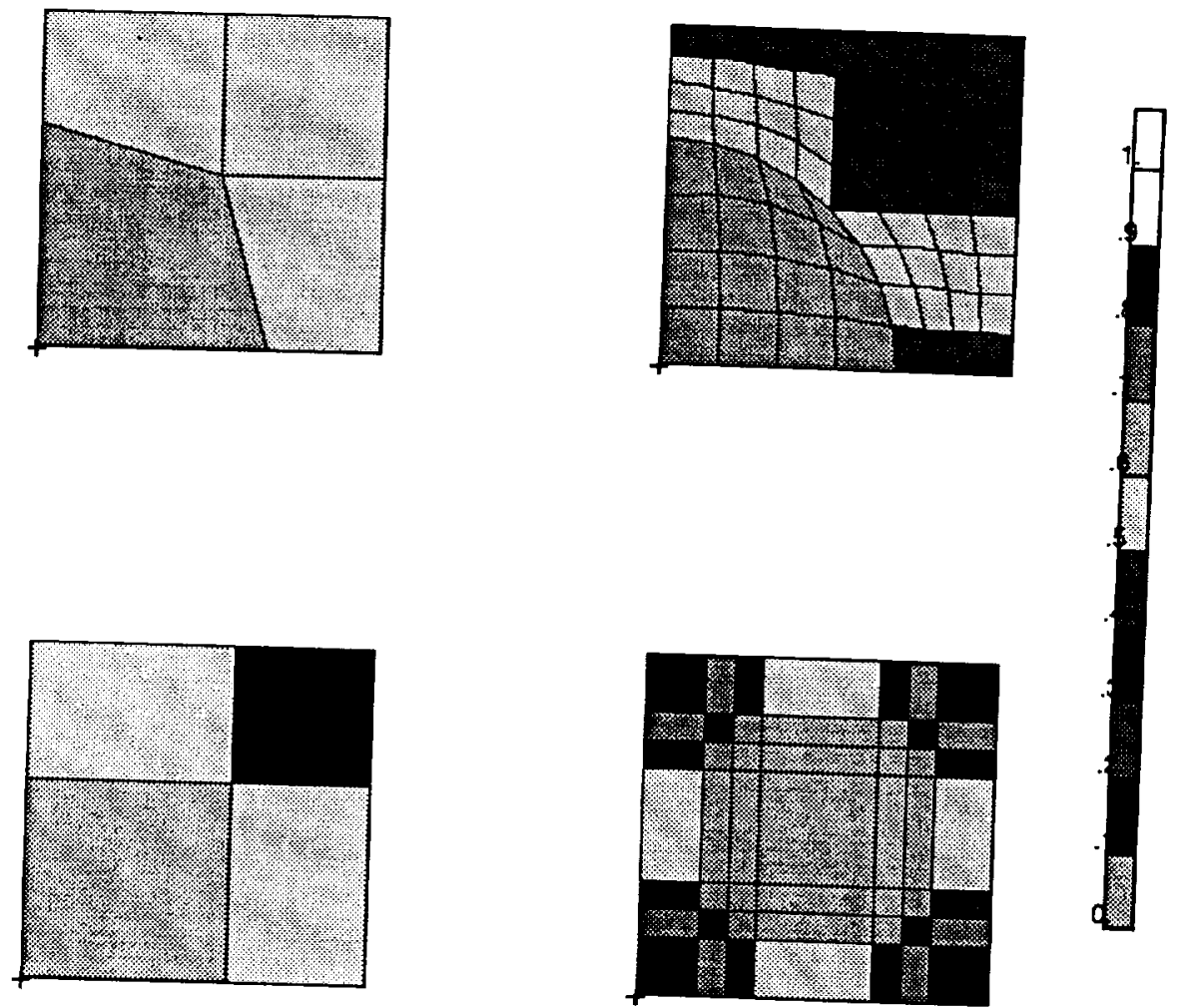

FIG. II-Representative damage states: finite element and GMC unit cells.

experimental results are currently available. However, full-scale burst pressure and fatigue tests will be performed on similar cladded MMC rings. Once these test results become available, a and continuum fatigue damage model

With regards to the micromechanics

dicting the transverse fatigue behavior fatigue damage analysis, future work will involve prealso be made between the finite element the same SiC/Ti-15-3 composite. Comparisons will of accuracy and computational efficiency. Finally, an atteneralized method of cells in terms based MMC ring insert life analysis, but this time an attempt will be made to repeat the macro-

\section{References}

(1) Arnold, S. M. and Kruch, S., "Differential Continuum Damage Mechanics Models for Creep and Fatigue of Unidirectional Metal Matrix Composites," Intermational Journal of Damage Mechanics.
Vol. 3, No. 2, 1994, pp. 170-191. 12] Lemaitre, J. and Chaboche, J. L., Mechanics of Solid Materials, Cambridge University Press. Cam-
bridge, UK, 1990. [3] Chaboche, J. L.. "Continuum Damage Mechanics: Part I-General Concepts," Journal of Applied
Mechanics, Vol. 55, 1988, pp. 59-64.

14] Chaboche, J. L.. "Continuum Damage Mechanics: Part II-Damage Growth. Crack Initiation and Crack Growth, Journal of Applied Mechanics, Vol. 55, 1988, pp. 65-72. 
[5] PATRAN User Manual, The MacNeal-Schoendler Corp.. Los Angeles, CA, 1995.

6) MARC. Revision K 5, Volume D: User Subroutines, MARC Analysis Research Corporation, Palo Alto, CA. 1995.

17] Arnold S. M and Wilt T. E. "A Deformation and Life Prediction of a Circumferentially Reinforced SiCrTi 15-3 Ring," Reliability, Stress Analysis and Failure Prevention, DE-Vol. 55, R. J. Schaller, Ed. 1993, pp. 231-238.

$18 \mid$ Wilt. T. E. and Arnold, S. M., "A Computationally-Coupled Deformation and Damage Finite Element Methodology," HITEMP Review 1993, Vol II, NASA CP19117, NASA, Cleveland, OH, pp. $35: 1-15$.

[9] Paley, M. and Aboudi, J., "Micromechanical Analysis of Composites by the Generalized Cells Model, "Mechanics of Materials. Vol. 14, 1992, pp. 127-139

[10] Wilt, T. E. and Arnold, S. M., Micromechanics Analysis Code (MAC) User Guide: Version 2.0, NASA TM-107290. NASA, Cleveland, OH, 1996.

11] Wilt. T. E., "On the Finite Element Implementation of the Generalized Method of Cells Micromechanics Constitutive Model," NASA CR-195451, NASA, Cleveland, OH, 1995. 

$\because$ 